\title{
Lithium-Ion Battery Capacity Prediction Using Recursive Least Squares with Forgetting Factor
}

\author{
Z.W. Zhou \\ Science and Technology on Reliability Physics and \\ Application of Electronic Component Laboratory, \\ Guangzhou, China \\ China Electronic Product Reliability and Environmental \\ Testing Research Institute, Guangzhou, China
}

\author{
Y. Huang \\ Science and Technology on Reliability Physics and \\ Application of Electronic Component Laboratory, \\ Guangzhou, China \\ China Electronic Product Reliability and Environmental \\ Testing Research Institute, Guangzhou, China
}

\author{
Y.D. $\mathrm{Lu}$ \\ Science and Technology on Reliability Physics and \\ Application of Electronic Component Laboratory, \\ Guangzhou, China \\ China Electronic Product Reliability and Environmental \\ Testing Research Institute, Guangzhou, China
Z.Y. Shi
Science and Technology on Reliability Physics and Application of Electronic Component Laboratory, Guangzhou, China
China Electronic Product Reliability and Environmental Testing Research Institute, Guangzhou, China

\section{X. $\mathrm{Li}$}

China Electronic Product Reliability and Environmental Testing Research Institute, Guangzhou, China

\begin{abstract}
How to predict capacity for lithium-ion battery is one of the most important problems in the field of battery health management. To make the newest data more efficiently, this paper proposes recursive least squares with forgetting factor to estimate the coefficients of the linear capacity degradation model, and presents the adaptive capacity prediction based on the estimation result. The experiment example demonstrates the effectiveness of the proposed approach
\end{abstract}

Keywords-lithium-ion battery; capacity prediction; recursive least squares with forgetting factor; linear degradation model

\section{INTRODUCTION}

Much effort has been concentrated on the prognostics and health management (PHM) technology for lithium-ion battery[1,2,3], since the PHM plays an vital role in the battery management system. There are two exquisite technology problems in the implementation of BMS, i.e., state-of-charge(SOC) estimation/prediction and remaining useful life (RUL) prediction. The SOC estimation is to determine when the battery arrives the end-of-discharge time during one charge-discharge cycle, and the RUL prediction aims to explain how many charge-discharge cycles before the battery reaches the capacity threshold. This paper focuses on the capacity degradation process modelling and its related prediction problem.

Generally speaking, there are three main ways to build the capacity degradation process under constant current and constant temperature. The first one is to build the degradation process by electronic-chemical analysis. A remaining useful life prediction model is presented to explain that the reduction in active reactant and lithium-ion is the main cause of capacity reduction in [4]. However, it is time-consuming to build the electronic-chemical model to describe the capacity degradation process. The second way is to build the model by use of data-driven method. For example, the double exponential model is used to describe the degradation curve, Dempster-Shafer is introduced to determine the unknown model parameters, and the particle filtering algorithm is employed to predict the RUL in [5]. The prediction precision of Auto-regression integrated movingaverage model (ARIMA), extended Kalman filtering, supported vector machine (SVM) and particle filtering (PF) is compared in [6]. The third way to deal with RUL prediction problem is to combine the data-driven methods with electronic-chemical method. For instance, a particle filtering framework is given to predict the RUL based on the empirical model that is described by a nonlinear state-space model in $[7,8]$.

When the lithium-ion battery discharges in constant current under constant temperature, the degradation curve behaves smoothly. Therefore, it is appropriate to describe the degradation in a simple way. Moreover, it is difficult to fit the degradation curve by one constant model in the whole cycle life, since the battery has very long cycle life.

For these two reasons, our paper aims to build the degradation curve by use of a slowly time-varying linear model, and predict remaining useful life in a data-driven way. To make the newest data more efficiently, this paper proposes recursive least squares with forgetting factor to estimate the coefficients of the linear capacity degradation model, and presents the adaptive capacity prediction based on the estimation result. 
The rest of the paper is organized as follows. The prediction problem statement is presented in section 2, the recursive least squares with forgetting factor algorithm is given in section 3, and the experiment example is demonstrated in section 4. Some concluding remarks are given in section 5 .

\section{PROBLEM STATEMENT}

When the lithium-ion battery discharges with constant current under constant temperature, the linear model is widely used to describe the capacity degradation process for its simplicity and low computational load. To be more specific, the linear degradation model is as follows

$$
C_{n}=a n+b+e_{n},
$$

where $a, b$ are the unknown constant model parameters and $e_{n}$ is the capacity measurement error that arises from measurement noise or model error.

The eqn. (1) shows that the lithium-ion capacity degrades linearly when the charge-discharge cycle increases. To build the model (1), it is equivalent to solve the optimization problem

$$
\min _{a, b}\left\{\sum_{n=1}^{N}\left(C_{n}-a n-b\right)^{2}\right\}
$$

To solve the minimization problem (2), the expression for (1) can be presented in the matrix form based on capacity measurement data $\left\{C_{1}, C_{2}, \ldots, C_{N}\right\}$ as follows

$$
\left(\begin{array}{c}
C_{1} \\
C_{2} \\
\ldots \ldots \\
C_{N}
\end{array}\right)=\left(\begin{array}{cc}
1 & 1 \\
2 & 1 \\
\ldots & \ldots \\
N & 1
\end{array}\right)\left(\begin{array}{l}
a \\
b
\end{array}\right)+E_{N}
$$

Let

$$
Y_{N}=\left(\begin{array}{c}
C_{1} \\
C_{2} \\
\ldots \\
C_{N}
\end{array}\right), A_{N}=\left(\begin{array}{cc}
1 & 1 \\
2 & 1 \\
\ldots & \ldots \\
N & 1
\end{array}\right), E_{N}=\left(\begin{array}{c}
e_{1} \\
e_{2} \\
\ldots \\
e_{N}
\end{array}\right), \theta=\left(\begin{array}{l}
a \\
b
\end{array}\right)
$$

then we get the unknown parameters estimation by least squares algorithm

$$
\theta_{N}=\left(\begin{array}{c}
\hat{a}_{N} \\
\hat{b}_{N}
\end{array}\right)=\left(A_{N}^{T} A_{N}\right)^{-1} A_{N}^{T} Y_{N} .
$$

Based on the estimation result, one-step capacity prediction can be expressed by

$$
\hat{C}_{N+1}=\hat{a}_{N} *(N+1)+\hat{b}_{N}
$$

Moreover, we can obtain the remaining useful life (RUL) can be given as follows

$$
R U L_{N}=\max \left\{k \mid \hat{C}_{N+k}=\hat{a}_{N} *(N+k)+\hat{b}_{N}>C_{\text {threshold }}\right\}
$$

It means that if $\hat{C}_{N+k}$ firstly decreases to the threshold $C_{\text {threshold }}$, then charge-discharge cycle RUL is defined as the remaining useful life.

Although the least squares algorithm is very easy to implement, the linear model cannot describe the capacity degradation trend during its whole cycle life. For example, we consider the capacity degradation data No. 45 battery that presented on NASA website.

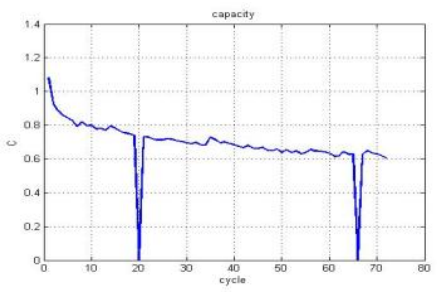

FIGURE I. CAPACITY DEGRADATION DATA.

Fig.1 shows that the No.45 battery capacity has sharp degradation in the first several charge-discharge cycles under temperature 4 degree, and the capacity degenerates gradually after about 10 cycles. This phenomenon implies that it is difficult to describe the capacity degradation trend by constant linear model even under constant discharge current and constant work temperature.

For this reason, this paper aims to present a slowly timevarying linear model to describe the lithium-ion battery capacity degradation, to identify the slowly time-varying parameters by recursive least squares with forgetting factor, and to derive the adaptive capacity prediction. It is expected that the proposed algorithm not only inherits the simplicity from least squares algorithm, but also has good precision in modelling capacity degradation..

\section{RECURSIVE LEAST SQUARES WITH FORGETTING FACTOR}

To be beginning, we make use of the slowly time-varying linear model to describe the capacity degradation

$$
C_{n}=a_{n} n+b_{n}+\varepsilon_{n},
$$

where $a_{n}, b_{n}$ are unknown time-varying parameters, $\varepsilon_{n}$ is the capacity measurement noise or modelling error.

To identify the model parameters $a_{n}, b_{n}$, we consider the following optimization problem

$$
\min _{a, b}\left\{\sum_{n=1}^{N} \lambda^{N-n}\left(C_{n}-a n-b\right)^{2}\right\} .
$$

The eqn. (4) shows that the data is forgotten with exponential velocity, i. e., the new data is give more weight and the old data is given less weight in the unknown parameter estimation. 
Denote $\varphi_{n}=\left(\begin{array}{l}n \\ 1\end{array}\right), \theta_{n}=\left(\begin{array}{l}a_{n} \\ b_{n}\end{array}\right)$, we obtain the least squares estimation with forgetting factor by derivation

$$
\hat{\theta}_{N}=\left(\sum_{n=1}^{N} \lambda^{N-n} \varphi_{n} \varphi_{n}^{T}\right)^{-1}\left(\sum_{n=1}^{N} \lambda^{N-n} \varphi_{n} C_{n}\right)
$$

In what follows we deduce the recursive form for least squares with forgetting factor. Denote

$$
\begin{gathered}
P_{N}=\left(\sum_{n=1}^{N} \lambda^{N-n} \varphi_{n} \varphi_{n}^{T}\right)^{-1}, \\
\hat{\theta}_{N-1}=P_{N-1}\left(\sum_{n=1}^{N-1} \lambda^{N-1-n} \varphi_{n} C_{n}\right),
\end{gathered}
$$

then it follows that

$$
P_{N}^{-1}=\lambda \sum_{n=1}^{N-1} \lambda^{N-1-n} \varphi_{n} \varphi_{n}^{T}+\varphi_{N} \varphi_{N}^{T}
$$

As a result, we can obtain

$$
\begin{aligned}
& \hat{\theta}_{N}=P_{N}\left(\sum_{n=1}^{N} \lambda^{N-n} \varphi_{n} C_{n}\right)=P_{N}\left(\lambda P_{N-1}^{-1} \hat{\theta}_{N-1}+\varphi_{N} C_{N}\right) \\
& =P_{N}\left(\left(P_{N}^{-1}-\varphi_{N} C_{N}\right) \hat{\theta}_{N-1}++\varphi_{N} C_{N}\right) \\
& =\hat{\theta}_{N-1}+P_{N} \varphi_{N}\left(C_{N}-\varphi_{N}^{T} \hat{\theta}_{N-1}\right) .
\end{aligned}
$$

Let $K_{N}=P_{N} \varphi_{N}$, and

$$
P_{N}=\frac{1}{\lambda}\left(P_{N-1}-\frac{P_{N-1} \varphi_{N} \varphi_{N}^{T} P_{N-1}}{\varphi_{N}^{T} P_{N-1} \varphi_{N}+\lambda}\right) .
$$

Then the recursive least squares with forgetting factor

$$
\hat{\theta}_{N}=\hat{\theta}_{N-1}+K_{N}\left(C_{N}-\varphi_{N}^{T} \hat{\theta}_{N-1}\right)
$$

where

$$
K_{N}=\frac{P_{N-1} \varphi_{N}}{\varphi_{N}^{T} P_{N-1} \varphi_{N}+\lambda}, P_{N}=\frac{1}{\lambda}\left(P_{N-1}-\frac{P_{N-1} \varphi_{N} \varphi_{N}^{T} P_{N-1}}{\varphi_{N}^{T} P_{N-1} \varphi_{N}+\lambda}\right) .
$$

Generally speaking, the forgetting factor can be chosen in the interval $\lambda \in[0.90,0.99]$. When the forgetting factor takes large weight such as 0.99 , it means that the past data is forgotten in low velocity. Otherwise, the past data is forgotten in high velocity.

There are two advantages in building linear model for capacity degradation. For one thing, the eqn. (5) can deal with the time-varying unknown parameter estimation problem. With the technology development of industrial manufacture and new material, the performance for lithiumion such as charge-discharge cycle life, energy, power and so on is improved evidently. Consequently, to describe the lithium-ion battery degradation process by one constant model within the whole charge-discharge cycle maybe employ some modelling error. The recursive least squares with forgetting factor can track the slowly time-varying parameters, i.e., the linear degradation model for lithium-ion capacity is built in a slowly time-varying way.

For another, from the expression for recursive least squares with forgetting factor (5), the new estimation $\hat{\theta}_{N}$ for $\theta_{N}=\left(\begin{array}{l}a_{N} \\ b_{N}\end{array}\right)$ can be achieved by the former one $\hat{\theta}_{N-1}$ and the revised new information, where the new information is defined as $C_{N}-\varphi_{N}^{T} \hat{\theta}_{N-1}$. Therefore, it can reduce the computational load in modelling process efficiently.

Based on the recursive estimation result, one-step capacity prediction can also be expressed by

$$
\hat{C}_{N+1}=\hat{a}_{N} *(N+1)+\hat{b}_{N} .
$$

Moreover, we can also obtain the remaining useful life (RUL) can be given by recursive least squares with forgetting factor algorithm as follows

$R U L_{N}=\max \left\{k \mid \hat{C}_{N+k}=\hat{a}_{N} *(N+k)+\hat{b}_{N}>C_{\text {threshold }}\right\}$, it means that if $\hat{C}_{N+k}$ firstly decreases to the threshold $C_{\text {threshold }}$, then charge-discharge cycle RUL is defined as the remaining useful life.

\section{EXPERIMENT EXAMPLE}

In what follows, we consider the capacity degradation data No.45 battery that presented on NASA website. For more details, please refer to http://ti.arc.nasa.gov/tech/dash/pcoe/prognostic-datapository/.

\section{A. Data pre-Processing}

The capacity degradation data for No.45 battery is showed in fig.1. It can be seen that there are two abnormal values during the charge-discharge cycle 22 and cycle 68 . To deal with the abnormality, we replace the capacity value of cycle 20 with the average capacity value of cycle 21 and cycle 23 . We also take the similar way to deal with the capacity value of cycle 68 .

\section{B. Parameters Estimation}

For the No. 45 battery, we make use of the recursive least squares with forgetting factor ( $\lambda=0.95$ ) algorithm to estimate the unknown parameters $a_{n}, b_{n}$. The estimation result is showed in fig.2.
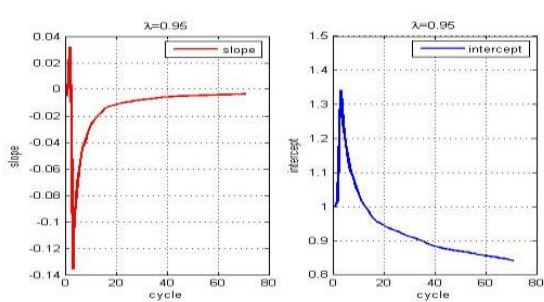

FIGURE II. UNKNOWN PARAMETER ESTIMATION. 
Fig.2 demonstrates that the slope estimation (in left subplot) and the intercept estimation (in right subplot) vary in slow way after about 10 charge-discharge cycle.

\section{One-step Capacity Prediction}

The one-step capacity prediction for lithium-ion battery is given in fig. 3 as follows,
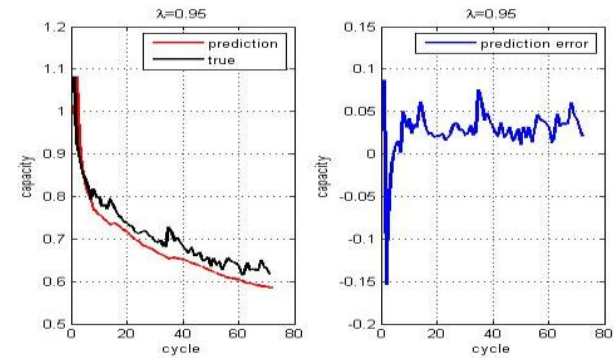

FIGURE III. ONE-STEP CAPACITY PREDICTION.

where black line (in the left subplot) denotes the capacity measurement value, and the red line (in the left subplot) denotes the one-step capacity prediction value. Moreover, the one-step capacity prediction error is showed in the right subplot. It can be deduced that the proposed recursive least squares with forgetting factor has good precision in predicting the capacity by one-step.

\section{Adaptive Remaining Useful Life Prediction}

The adaptive remaining useful life prediction is derived as follows: we make use of the first 20 capacity data, then we obtain the estimation result $\hat{a}_{20}, \hat{b}_{20}$ by recursive least squares with forgetting factor, therefore we derive the remaining useful life prediction $R U L_{20}=10$. Similarly, we make use of the first 21 capacity data, then we obtain the estimation result $\hat{a}_{21}, \hat{b}_{21}$ by recursive least squares with forgetting factor, therefore we derive the remaining useful life prediction $R U L_{21}=9$. So on and so forth. The adaptive remaining useful life prediction is showed in fig.4.

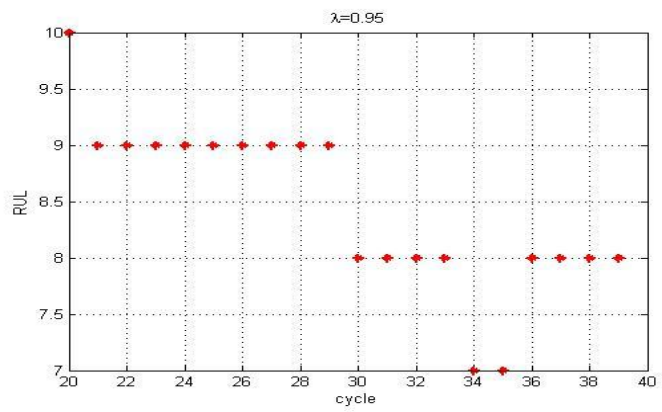

FIGURE IV. ADAPTIVE REMAINING USEFUL LIFE PREDICTION.

\section{REFERENCES}

[1] Goebel, K., Saha, B., Saxena, A., et al., Prognostics in battery health management. IEEE Instrumentation and Measurement Magazine, 11(4), pp. 33-40, 2008.

[2] Saha, B., Koshimoto, E., Quach, C.C., et al, Battery health management system for electric UAVs. Proc. of Aerospace Conference, pp.1 -9., 2011.
[3] Mohammad, R., Mohammed, A. A., Seungchul, Lee., et al, A comparative analysis of techniques for electric vechicle battery prognostics and health management, SAE international,11CV-0191, 2011 .

[4] Q. Zhang, \& White, R. E., Calendar life study of Li-ion pouch cells. Journal of Power Sources, 173, pp. 990-997, 2007.

[5] W. He, Williard, N., Osterman, M., et al, Prognostics of lithium-ion batteries based on Dempster-Shafer theory and the Bayesian Monte Carlo method. Journal of Power Sources, 196(23), pp. 10314-10321, 2011.

[6] Saha, B., Goebel, K., \& Christophersen, J., Comparision of Prognostic Algorithms for Estimating Remaining Useful Life of Batteries, Transactions of the Royal UK Institute on Measurement and Control, 31(3-4), pp.293-308, 2009.

[7] Saha, B. \& Goebel, K., Modeling Li-ion battery capacity depletion in a particle filtering framework, Annual Conference of the Prognostics and Health Management Society, pp. 1-10, 2009.

[8] Saha, B. \& Goebel, K., Model Adaption for Prognostics in a Particle Filtering Framework. International Journal of Prognostics and Health Management, 6, pp.1-10, 2011. 